\title{
Anomalous negative resistance in superconducting vanadium nanowires
}

\author{
J. Jorritsma* and J. A. Mydosh \\ Kamerlingh Onnes Laboratory, Leiden University, P.O. Box 9504, 2300 RA Leiden, The Netherlands
}

(Received 1 March 2000)

\begin{abstract}
Low-temperature electrical transport measurements were performed on large arrays of 150-nm-wide superconducting vanadium $(\mathrm{V})$ wires covered with either a thin layer of $\mathrm{Au}(\mathrm{V} / \mathrm{Au})$ or $\mathrm{Fe}(\mathrm{V} / \mathrm{Fe})$. The measurements were conducted using various electrical contact geometries. Our results show that a particular arrangement of the electrical contacts in combination with the superconducting proximity effect can result in a pronounced "negative resistance" anomaly below the resistive transition. We demonstrate that this "negative resistance" can be clearly reproduced by constructing resistor circuits based upon the particular contact arrangement.
\end{abstract}

\section{INTRODUCTION}

For many years, the effect of dimensionality on the electronic properties of superconductors has been a subject of extensive research. In particular, the role of disorder on the superconductivity in thin films and narrow wires is, even today, an active area of experimental and theoretical study. Various experimental investigations of (disordered) superconducting aluminum thin films ${ }^{1,2}$ and narrow wires ${ }^{3-6}$ report anomalous resistance peaks in the vicinity of the transition temperature $T_{c}$, where the resistance can exceed its normal-state value by as much as $30 \%$. These experimental results could be qualitatively understood by the formation of phase-slip centers or the presence of normal-superconducting interfaces ${ }^{1,2}$ between the two voltage probes of the sample. Recently, it was shown by radio-frequency irradiation or by applying sufficiently high dc driving currents that these phase-slip centers nucleate at particular spots in the sample with a locally reduced $T_{c} \cdot{ }^{6}$ Moreover, it was demonstrated that a resistance peak could also be induced by deliberately applying an ac noise current to narrow $\mathrm{Al}$ lines in addition to the ordinary probe current. ${ }^{5}$

Other experiments report a reduction of $T_{c}$ accompanied by a broadening of the resistive transition by narrowing the width of superconducting wires. ${ }^{7}$ Similar features were observed in narrow uniform $\mathrm{Pb}$ wires for which the width and thickness were systematically varied. ${ }^{8}$ The observed behavior was explained in terms of disorder-enhanced superconducting fluctuations near $T_{c}$. Below $T_{c}$ these fluctuations enhance the number of thermally activated phase slips, causing a resistance increase, whereas above $T_{c}$ such fluctuations lead to a resistance decrease. In later experiments the resistance of $\mathrm{Pb}$ wires similar to those of Sharifi et al. ${ }^{8}$ was studied in the presence of a magnetic field. ${ }^{9}$ These wires exhibit a negative magnetoresistance (MR) below $T_{c}$ within the transition region. Both the magnitude of the negative $\mathrm{MR}$ and the crossover field to positive MR increase with decreasing $T_{c}$ and wire cross section. The negative MR corresponds to an enhancement of superconductivity and a suppression of superconducting fluctuations by the magnetic field with respect to the zero-field case.

The transition from a strongly localized ("insulating') to a weakly localized ("metallic") state was systematically studied by Herzog et al. ${ }^{10}$ in various narrow superconducting and normal metal granular wires. When the thickness of these wires was slowly increased, a large abrupt drop in their resistance was observed. These results were speculated to be due to a first-order electronic phase transition between the above states. The size of the resistance gap was found to depend on the number of grains across the wire width and not on the specific material. This resistance gap decreases as the number of grains increases.

In this paper we present the results of low-temperature electrical transport measurements conducted on large arrays $\left(\sim 10^{4}\right)$ of narrow vanadium $(\mathrm{V})$ wires of $150 \mathrm{~nm}$ in width. Our initial purpose was to investigate the so-called proximity effect in these very narrow superconducting wires. In previous work, the proximity effect was studied in V/Fe multilayers, in which a suppression of $T_{c}$ was observed due to the presence of ferromagnetic Fe spacer layers. ${ }^{11,12}$ Extending this work, our $\mathrm{V}$ wires were covered with a thin layer of either ferromagnetic $\mathrm{Fe}(\mathrm{V} / \mathrm{Fe})$ or nonmagnetic $\mathrm{Au}(\mathrm{V} / \mathrm{Au})$. However, in the course of our investigation it turned out that our samples exhibited variable $T_{c}$ values, which prevented a systematic study of the proximity effect.

Nevertheless, electrical transport measurements performed on our $\mathrm{V} / \mathrm{Au}$ and $\mathrm{V} / \mathrm{Fe}$ wires reveal a very pronounced "negative resistance" below $T_{c}$. Previously, negative resistances were also observed in planar tunnel junctions when the junction resistance was smaller than the lead resistances in a four-terminal configuration. ${ }^{13-16}$ In this case, the negative resistance is an artifact caused by a nonuniform current distribution over the junction area. More recently, a negative resistance was observed in superconducting granular $\mathrm{Sn}$ wires of approximately the same width $(\sim 100 \mathrm{~nm})$ as our wires. ${ }^{17}$ In addition to reproducible MR oscillations, which were attributed to screening currents induced by magnetic flux threading phase coherent loops of grains, the MR of these wires also displayed a clear negative resistance below $T_{c}$. Here it was speculated that this negative resistance is caused by screening currents in the narrow voltage probes distorting the current path, but the exact cause is still unclear. We therefore proceeded to explore this striking negative resistance in our narrow $\mathrm{V} / \mathrm{Au}$ and $\mathrm{V} / \mathrm{Fe}$ wires rather than continuing to investigate the proximity effect. Our electrical transport measurements were performed using three different electrical contact geometries (see below). Our results show that the negative resistance anomaly below $T_{c}$ is induced by 
a particular arrangement of the electrical contacts in combination with the superconducting proximity effect. By constructing passive resistor circuits based upon the contact geometry, we will demonstrate that this negative resistance anomaly can be clearly reproduced. Hence, we require no new or exotic physics to explain the negative resistance observed in our narrow wires.

\section{EXPERIMENT}

Our V/Au and $\mathrm{V} / \mathrm{Fe}$ wires were prepared by oblique electron-beam evaporation onto V-groove-patterned InP substrates with a period of $240 \mathrm{~nm} .{ }^{18}$ The V, Au, and Fe were evaporated at a rate of 0.2 and $0.1 \mathrm{~nm} / \mathrm{s}$, respectively, in a vacuum system with a base pressure of $\sim 10^{-8}$ mbar. All targets were casted in an arc furnace from $99.99 \%$ pure bulk material. During evaporation, the V-groove InP substrates were held at room temperature. The width of the wires was $\approx 150 \mathrm{~nm}$ as determined from inspection with a scanning electron microscope.

Films with the same layer composition as the wires were deposited simultaneously onto flat $\mathrm{Si}(001)$ substrates to ensure the same preparation conditions. These simultaneously evaporated films served as reference samples for calibrating the thickness of the $\mathrm{V}, \mathrm{Au}$, and $\mathrm{Fe}$ layers using electronprobe microanalysis. The thickness of the $\mathrm{V}$ layer is $\approx 40$ $\mathrm{nm}$, whereas that of the Au and Fe layers is $\approx 2 \mathrm{~nm}$. The crystalline structure of the films was analyzed using x-ray diffraction (XRD). The XRD analysis indicated that the films possess a nanocrystalline structure with a random orientation of the crystallographic axes of constituting grains. The lattice constant of the $\mathrm{V}$ layers was the same as that for bulk vana$\operatorname{dium}(0.302 \mathrm{~nm})$.

Magnetization measurements conducted in the normal state at $20 \mathrm{~K}$ using a superconducting quantum interference device (SQUID) magnetometer (Quantum Design MPMS$5 \mathrm{~S}$ ) confirmed that the thin $\mathrm{Fe}$ cover layer is ferromagnetic with a moment of $\mu_{\mathrm{Fe}} \approx 1.1 \mu_{B} / \mathrm{Fe}$ atom.

Electrical transport experiments were performed in a fourprobe configuration using the three electrical contact arrangements shown in Fig. 1. The electrical contacts in geometry $A$ and $B$ were made on top of the wires by evaporating $300 \mathrm{~nm}$ of $\mathrm{Au}$ through a mechanical mask held in close proximity to the surface of the wire samples. The number of wires contacted in parallel is typically $\sim 5 \times 10^{3}$, while the distance between adjacent contact pads is $\approx 0.5 \mathrm{~mm}$. The wire samples were glued in a chip holder, after which the current and voltage leads ( $\mathrm{Au}, \sim 20 \mu \mathrm{m}$ in diameter) were attached to the $\mathrm{Au}$ contact pads by ultrasonic wire bonding. Contact geometry $B$ is the same as geometry $A$, but with the current and voltage leads interchanged. In contact geometry $C$ the current and voltage leads $(\mathrm{Cu}, \sim 100 \mu \mathrm{m}$ in diameter) are attached to the sample using either silver paint or indium pressure bonding. For all contact geometries, the contact resistance is less than $0.5 \Omega$.

Low-temperature electrical transport measurements were performed in various cryostats down to $1.4 \mathrm{~K}$ using ac or dc measuring techniques. All measurements yield the same results. The measurements were performed in external magnetic fields up to $8 \mathrm{~T}$ oriented parallel and normal to the sample plane. For resistance measurements the probe current
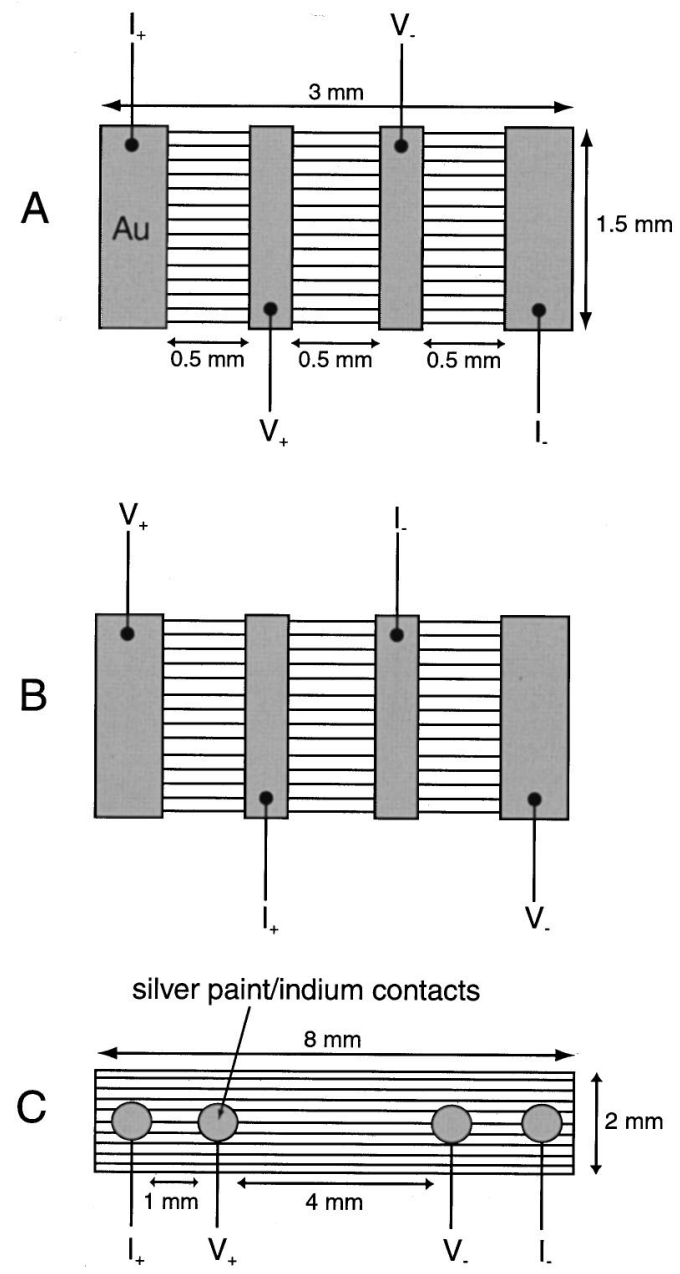

FIG. 1. Various electrical contact arrangements $(A, B$, and $C)$ used to perform electrical transport measurements on V/Au and $\mathrm{V} / \mathrm{Fe}$ wire samples.

density was kept as low a possible, to avoid heating effects, and was typically in the range of $10-100 \mathrm{~A} / \mathrm{cm}^{2}$. By varying the current density within this range, no change in the resistive transitions was observed. During dc measurements, the voltage signal was always corrected for thermovoltages by reversing the current direction or by recording the thermovoltage at zero measuring current. Both methods yield exactly the same results.

The electronic mean free path of the wires $l \approx 3 \mathrm{~nm}$ was estimated from their residual resistivity at $4.2 \mathrm{~K}$. Since this value is much smaller than the BCS coherence length $\xi_{0}$ $\approx 40 \mathrm{~nm}$ for pure bulk vanadium, ${ }^{19}$ our wire samples should be treated within the "dirty limit" regime. The dirty-limit Ginzburg-Landau coherence length $\xi(0) \approx 9 \mathrm{~nm}$ was determined from the change of $T_{c}$ in a fixed external magnetic field.

\section{RESULTS AND DISCUSSION}

Figure 2 illustrates the temperature dependence of the resistance (normalized to its normal-state value at $4.2 \mathrm{~K}$ ) for 150-nm-wide $\mathrm{V} / \mathrm{Au}$ and $\mathrm{V} / \mathrm{Fe}$ wires in the vicinity of $T_{c}$ in various fixed external magnetic fields applied normal to the sample plane. The measurements were performed using con- 

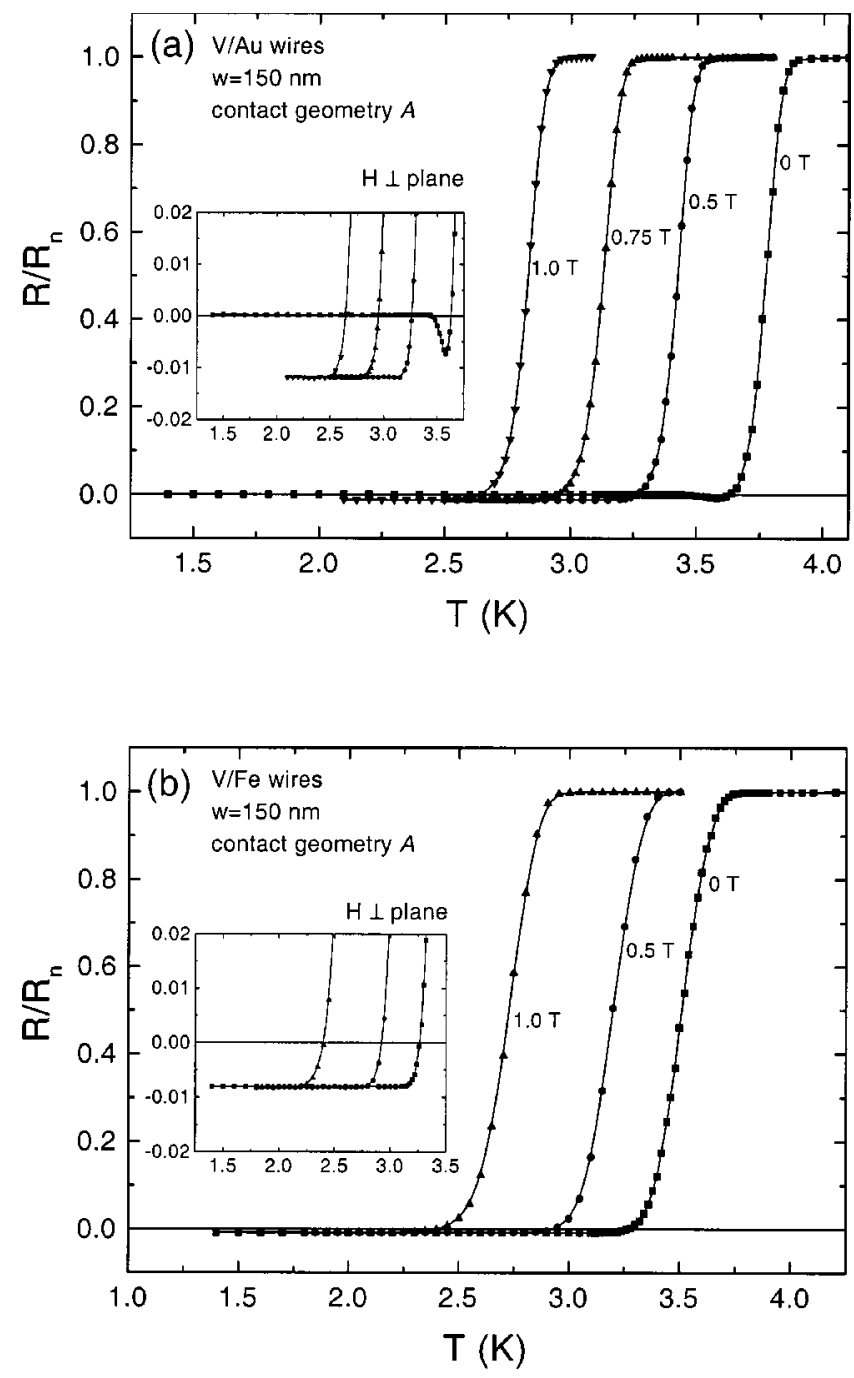

FIG. 2. The temperature dependence of the resistance in various fixed external fields applied normal to the sample plane near $T_{c}$ for (a) 150-nm-wide V/Au wires and (b) 150-nm-wide V/Fe wires. The measurements were performed using contact geometry $A$. Insets: magnification of the resistance curves below $T_{c}$. Apparent is the striking negative resistance which appears below $T_{c}$.

tact geometry $A$ (see Fig. 1). Very striking is the appearance of a "negative resistance" for these wires below $T_{c}$ [see insets in Figs. 2(a) and 2(b)]. For our V/Au wires in zero external field, this negative resistance is only present just below $T_{c}$ and disappears again at temperatures sufficiently below $T_{c}$. Nevertheless, in the presence of an external magnetic field, this negative resistance persists down to lowest temperatures with a value which is approximately independent of temperature and external field. Unlike the V/Au wires, the negative resistance even in zero field for our $\mathrm{V} / \mathrm{Fe}$ wires is approximately independent of temperature and persists down to the lowest temperatures. Application of an external magnetic field does not affect the temperature dependence or magnitude of the negative resistance. Hence, in the presence of an external field, the temperature dependence and magnitude $(\sim 1 \%$ of the normal-state resistance) of the negative resistance is approximately the same for our 150$\mathrm{nm}$-wide $\mathrm{V} / \mathrm{Au}$ and $\mathrm{V} / \mathrm{Fe}$ wires. We note that the behavior of this negative resistance as a function of temperature is independent of the way how the temperature is varied. Resistance measurements conducted using contact geometry $B$ yield similar results compared to those obtained for contact geometry $A$.

The negative resistance anomaly observed in the resistance measurements using contact geometries $A$ and $B$ is also manifested in $I-V$ curve measurements (not shown) by the appearance of a pronounced negative voltage below $T_{c} \cdot{ }^{20}$ We found that for small enough ac and dc driving currents and sufficiently low temperatures, no negative voltage is induced in our V/Au wires. However, for higher driving currents, a negative voltage appears which increases linearly with driving current. In contrast, our $\mathrm{V} / \mathrm{Fe}$ wires exhibit a negative voltage even for the smallest driving currents, which increases linearly with current. The linear current dependence of the negative voltage indicates that the negative resistance exhibits Ohmic behavior. The negative resistance derived from the $I-V$ curves equals the value observed in the resistance measurements.

In summary, by using contact geometries $A$ and $B$ our 150-nm-wide $\mathrm{V} / \mathrm{Au}$ and $\mathrm{V} / \mathrm{Fe}$ wires exhibit a pronounced negative resistance below $T_{c}$. The results for the V/Au wires show that in zero external field and low enough driving currents this negative resistance vanishes at temperatures sufficiently below $T_{c}$. However, the negative resistance reappears when a small external magnetic field or a higher driving current is applied to the wires. For the V/Fe wires the resistance is negative below $T_{c}$ and independent of temperature, magnetic field, and driving current.

Figure 3 displays the temperature dependence of the resistance for our $150-\mathrm{nm}$-wide $\mathrm{V} / \mathrm{Au}$ and $\mathrm{V} / \mathrm{Fe}$ wires in various fixed external fields oriented normal to the sample plane. The experiments were performed using contact geometry $C$ (see Fig. 1). In contrast to the measurements performed using contact geometry $A$ and $B$, no negative resistance anomaly is observed below $T_{c}$ for this contact arrangement. The values of $T_{c}$ equal those measured using contact geometries $A$ and $B$.

The results of our resistance measurements clearly indicate that the negative resistance anomaly is associated with a particular arrangement of the electrical contacts. We note that in contact geometries $A$ and $B$ the two current leads are connected to the Au contact pads near the sample edge, but on opposite sides of the multiwire sample. Hence, when a current is injected, part of it will flow through the contact pads from one edge to the other edge of the wire sample, which gives rise to an extra resistance in series with the resistance of the wires. Like the two current leads, the two voltage leads are attached to the Au contact pads near the sample edge on opposite sides of the wire sample. It is therefore conceivable that the two narrow voltage leads probe the electrochemical potential of two different sets of wires located on opposite sides of the wire sample. Hence, in the presence of a driving current, the Au voltage pads are not equipotential areas.

Based upon this qualitative analysis we can model contact geometry $A$ by the resistor circuit illustrated in Fig. 4(a), in order to allow a more quantitative analysis. In our analog resistor network two current paths or branches can be distinguished. Each current path corresponds to a set of wires probed by one of the voltage leads. Here $R_{1}, R_{2}, R_{3}, R_{6}$, $R_{7}$, and $R_{8}$ represent the resistances of the wire sections 

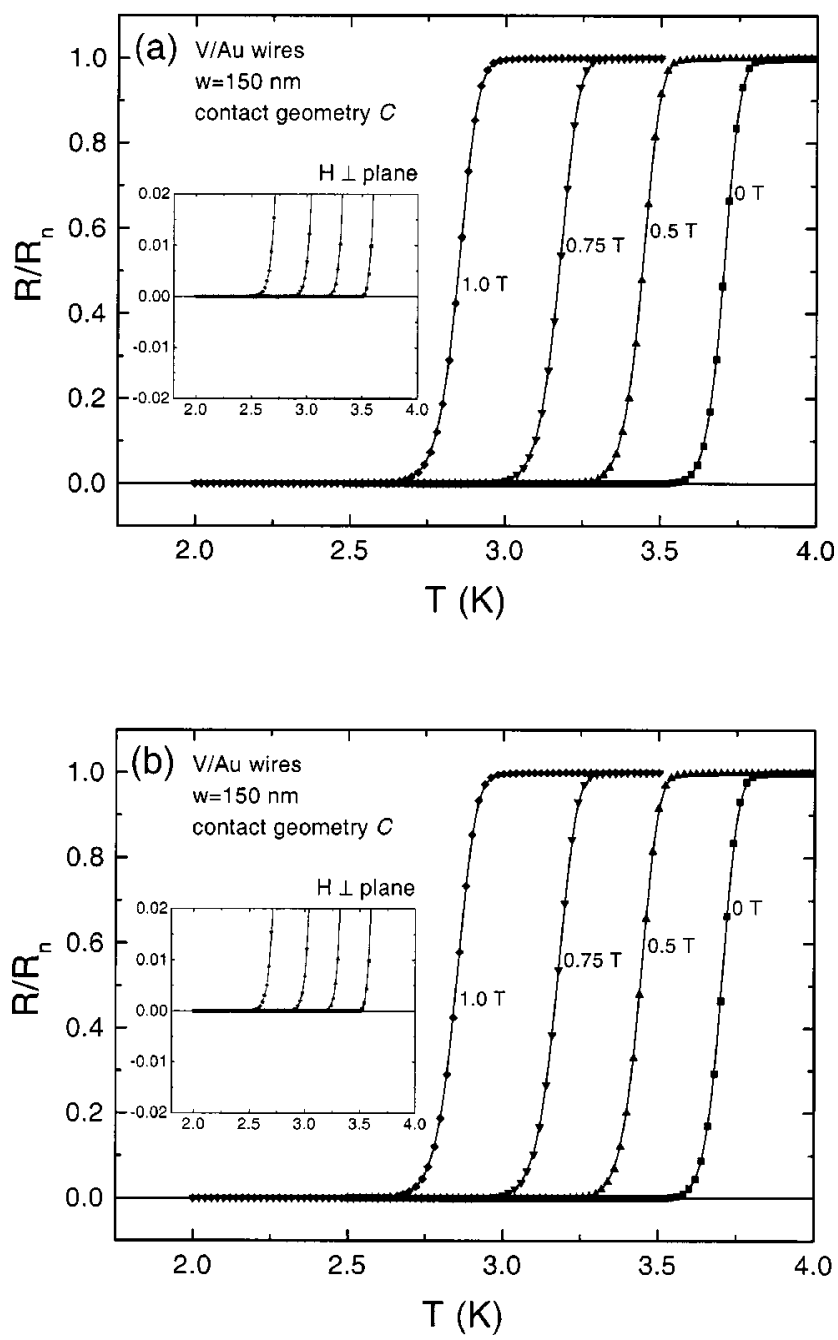

FIG. 3. The temperature dependence of the resistance in various fixed external fields oriented normal to the sample plane near $T_{c}$ for (a) 150-nm-wide V/Au wires and (b) $150-\mathrm{nm}$-wide V/Fe wires. The resistance was measured using contact geometry $C$. Insets: magnification of the resistance curves below $T_{c}$. Note the absence of the negative resistance below $T_{c}$ for this contact arrangement.

between adjacent contact pads, whereas $R_{4}, R_{5}$ and $R_{9}, R_{10}$ represent the resistances of the two Au current and voltage pads, respectively. Because the length of the wire sections between adjacent contact pads is the same in contact geometry $A$, we can simplify our analysis by assuming $R_{1}=R_{2}$ $=R_{3}=R_{6}=R_{7}=R_{8}$. Moreover, since the dimensions of the Au contact pads are approximately equal, we further assume that $R_{4}=R_{5}=R_{9}=R_{10}$. The "measured resistance" $R_{A}$ $=\left(V_{+}-V_{-}\right) / I$ for contact geometry $A$ expressed in terms of $R_{1}$ and $R_{4}$ is then given by

$$
R_{A}=\frac{2 R_{1}^{2}+2 R_{1} R_{4}-R_{4}^{2}}{4\left(R_{1}+R_{4}\right)} .
$$

From Eq. (1) it is easy to see that the measured resistance $R_{A}$ becomes negative whenever $R_{1} \ll R_{4}$, which occurs when the $\mathrm{V}$ wires becomes superconducting $\left(R_{1}=0\right)$, while the $\mathrm{Au}$ contact pads remain resistive $\left(R_{4}>0\right)$.

By using Eq. (1) we tried to reproduce the negative resistance anomaly observed below $T_{c}$ for our 150-nm-wide (a)

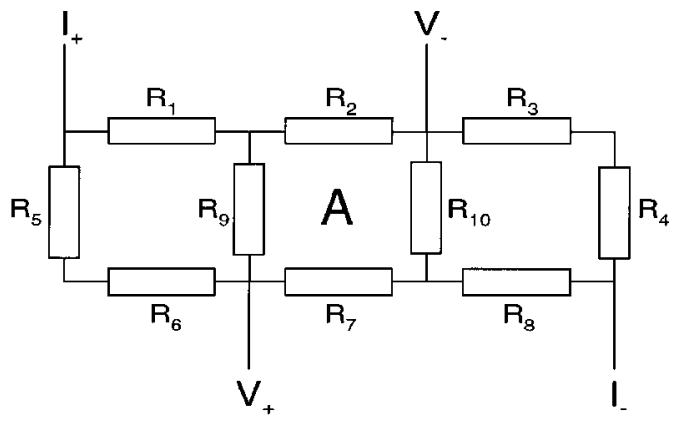

(b)

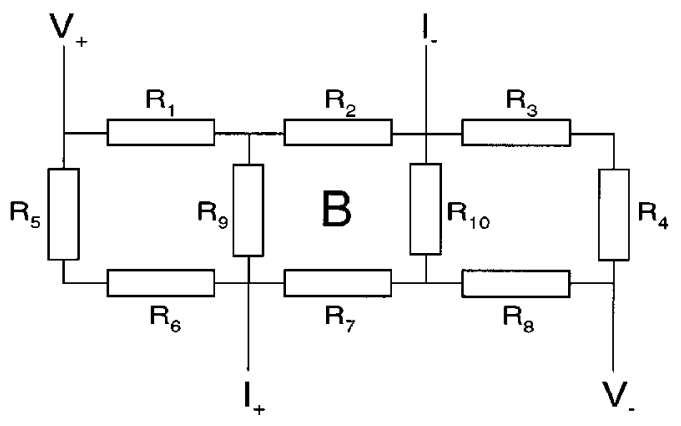

FIG. 4. The two resistor circuits corresponding to contact geometries $A$ and $B$ in Fig. 1.

$\mathrm{V} / \mathrm{Au}$ and $\mathrm{V} / \mathrm{Fe}$ wires. In the following we will illustrate this for the zero-field resistance versus temperature $[R(T)]$ curves of our $\mathrm{V} / \mathrm{Au}$ and $\mathrm{V} / \mathrm{Fe}$ wires measured using contact geometry $A$ (see Fig. 2). For the assumed temperature dependence of $R_{1}$ we take the experimental $R(T)$ curves of our $\mathrm{V} / \mathrm{Au}$ and $\mathrm{V} / \mathrm{Fe}$ wires measured in zero field using contact geometry $C$ (see Fig. 3), in which no negative resistance anomaly was observed.

In order to reproduce the experimental zero-field $R(T)$ curve of the V/Au wires measured using contact geometry $A$ [see Fig. 2(a)], we have to assume $R_{4}(T)=0.02 R_{1}(T)$ for $T>T_{c 1}$ and $T_{c 4}=0.93 T_{c 1}$. Here $T_{c 1} \approx 3.75 \mathrm{~K}$ represents the transition temperature of $R_{1}$ (i.e., V/Au wires), whereas $T_{c 4}$ represents the transition temperature of $R_{4}$ (i.e., Au contact pads). The assumed zero-field $R_{1}(T)$ and $R_{4}(T)$ curves are illustrated in Fig. 5(a). Substituting $R_{1}(T)$ and $R_{4}(T)$ into Eq. (1) we obtain the temperature dependence of $R_{A}$ $\left[R_{A}(T)\right]$ in zero field. The simulated zero-field $R_{A}(T)$ curve is shown in Fig. 5(c). Note that the negative resistance dip just below $T_{c}$ is clearly reproduced by this simulation.

Hence, in order to explain the negative resistance dip in the experimental zero-field $R(T)$ curve of our V/Au wires [see Fig. 2(a)], we have to assume that a superconducting layer is nucleated in the contact pads which extends over the whole area of the contact pads at $T_{c 4}=0.93 T_{c 1} \approx 3.49 \mathrm{~K}$. The only possible way to induce such a superconducting layer in the Au contact pads at this temperature is via the proximity effect. The proximity effect occurs because Cooper pairs from a superconductor in contact with a normal metal penetrate into the normal metal over a typical distance known as the proximity length. The proximity length in a normal metal $\xi_{n} \approx\left(\hbar D_{n} / 2 \pi k_{B} T\right)^{1 / 2}$, where $D_{n}=v_{F} l / 3$ is the diffusion constant, $v_{F}$ the Fermi velocity, and $l$ the elastic mean free path of the normal metal. Hence $\xi_{n}$ increases for decreasing temperature $T$.

Since the Au contact pads are deposited onto a regular 

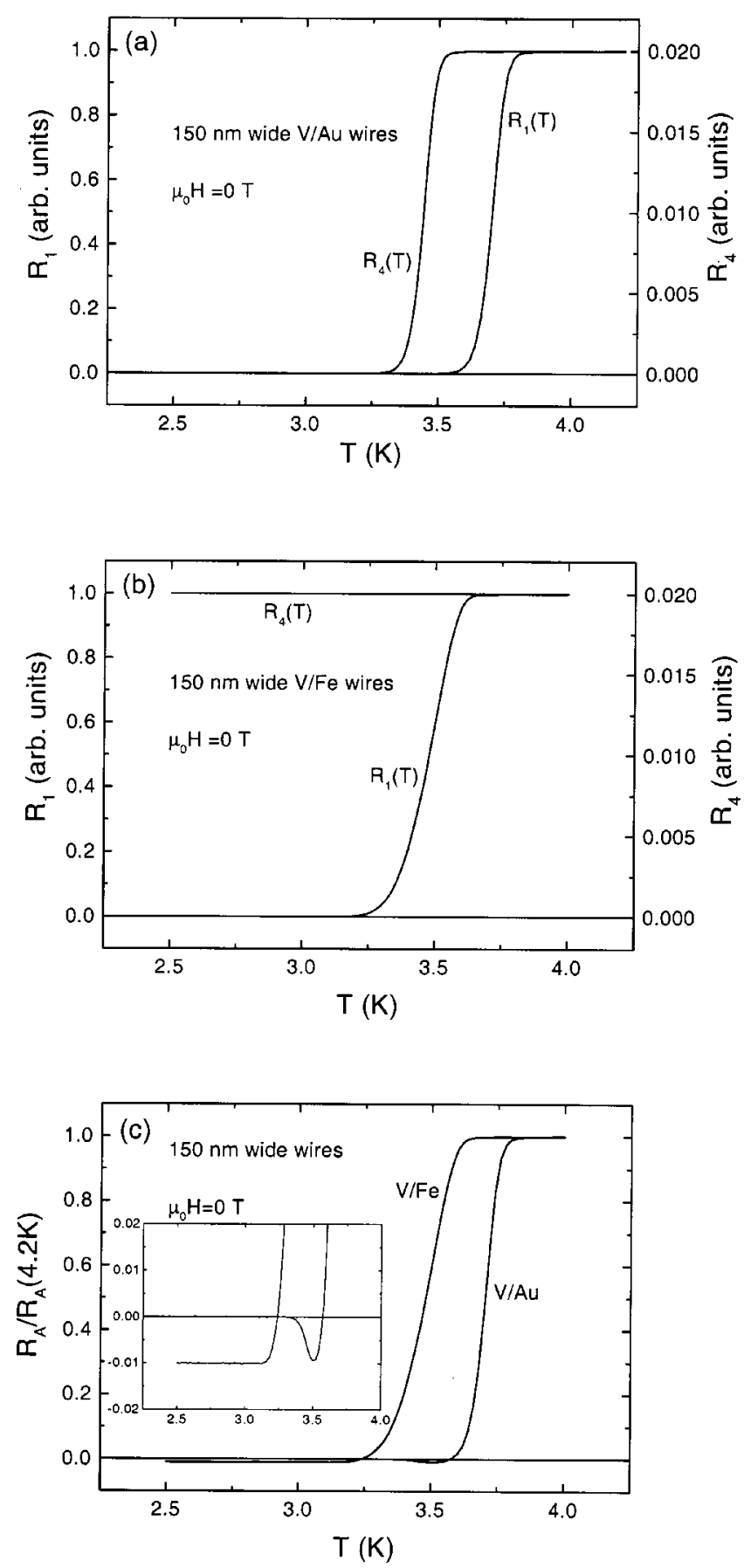

FIG. 5. The assumed temperature dependence of the resistances $R_{1}$ and $R_{4}$ of the resistor circuit corresponding to contact geometry $A$ in zero magnetic field for (a) 150-nm-wide V/Au wires and (b) 150-nm-wide V/Fe wires. (c) The simulated temperature dependence of the measured resistance $R_{A}$ (normalized to its value at 4.2 $\mathrm{K}$ ) in zero magnetic field for our $150-\mathrm{nm}$-wide V/Au wires [using $R_{4}(T)=0.02 R_{1}(T)$ for $T>T_{c 1}$ and $\left.T_{c 4}=0.93 T_{c 1}\right]$ and for our 150nm-wide $\mathrm{V} / \mathrm{Fe}$ wires [using $R_{4}(T)=0.02 R_{1}(T)$ for $T>T_{c 1}$ and $\left.T_{c 4} \ll T_{c 1}\right]$.

grid of V wires, which are only $240 \mathrm{~nm}$ apart, it is conceivable that, once the $\mathrm{V}$ wires have become superconducting, superconductivity is also induced (via the proximity effect) in regions of the contact pads surrounding the $\mathrm{V}$ wires. The suppression of the negative resistance anomaly in the zerofield $R(T)$ curve of our V/Au wires [see Fig. 2(a)] for de- creasing temperature reflects the growth of these proximityeffect-induced superconducting regions. At $T=T_{c 4}$ $\approx 3.49 \mathrm{~K}$, the superconducting volumes induced around each $\mathrm{V}$ wire start to overlap such that a continuous superconducting layer is formed, which extends over the whole area of the contact pads. In order to form a continuous superconducting layer, we estimate $\xi_{n} \sim \frac{240}{2} \mathrm{~nm}$, because the distance between neighboring wires is $240 \mathrm{~nm}$. By assuming $l \sim 100 \mathrm{~nm}$ for the Au contact pads and using $v_{F} \approx 1.4 \times 10^{6} \mathrm{~m} / \mathrm{s}$ for $\mathrm{Au}$, we find $\xi_{n} \sim 130 \mathrm{~nm}$ at $T=3.49 \mathrm{~K}$. This value is in good agreement with $\xi_{n} \sim 120 \mathrm{~nm}$ estimated to form a continuous superconducting layer extending over the whole area of the contact pad.

Resistance measurements performed on our V/Au wires using contact geometry $A$ show that the negative resistance persists down to the lowest temperatures by applying a small $(\sim 0.1 \mathrm{~T})$ external magnetic field. This behavior implies that in the presence of a small magnetic field, the Au contact pads do not become superconducting, but remain resistive $\left(R_{4}\right.$ $>0)$. Consequently, the measured resistance $R_{A}(T)$ remains negative when the V/Au wires are superconducting $\left(R_{1}\right.$ $=0$ ). The fact that the contact pads remain normal can be explained by assuming that nucleation of a superconducting layer is strongly suppressed by application of an external magnetic field of $\sim 0.1 \mathrm{~T}$.

In addition, we also tried to simulate the experimental zero-field $R(T)$ curve of our 150-nm-wide V/Fe wires measured using contact geometry $A$ [see Fig. 2(b)]. The experimental $R(T)$ curve can be reproduced by assuming $R_{4}(T)$ $=0.02 R_{1}(T)$ for $T>T_{c 1}$ and $T_{c 4} \ll T_{c 1}$. The assumed zerofield $R_{1}(T)$ and $R_{4}(T)$ curves are illustrated in Fig. 5(b). Figure 5(c) shows the simulated zero-field $R_{A}(T)$ curve based upon the assumed $R_{1}(T)$ and $R_{4}(T)$ curves. Also in this case, the experimental zero-field $R(T)$ curve of our $\mathrm{V} / \mathrm{Fe}$ wires, measured using contact geometry $A$, can be nicely reproduced. Since for this simulation we have to assume $R_{4}(T)=0.02 R_{1}(T)$ for $T>T_{c 1}$ and $T_{c 4} \ll T_{c 1}$, such implies that the contact pads remain normal $\left(R_{4}>0\right)$ for $T>T_{c 4}$. Hence, in zero external field, no proximity-effect-induced superconducting layer is formed in the contact pads of our $\mathrm{V} / \mathrm{Fe}$ wire samples. This can be explained by considering the fact that the thin ferromagnetic Fe layer on top of the $\mathrm{V} / \mathrm{Fe}$ wires breaks Cooper pairs, such that diffusion of Cooper pairs into the normal Au contact pads is strongly suppressed. Because the contact pads remain resistive $\left(R_{4}>0\right)$, the measured resistance $R_{A}$ in Eq. (1) will remain negative down to $T \approx T_{c 4} \ll T_{c 1}$.

The resistance measurements performed using contact geometry $A$ show that the zero-field $R(T)$ and field $R(T, H)$ curves of our $\mathrm{V} / \mathrm{Fe}$ wires exhibit the same temperature behavior (apart from the usual reduction of $T_{c}$ in an external magnetic field). This field-independent behavior can be understood by realizing that superconductivity in the contact pads is already strongly suppressed by the ferromagnetic $\mathrm{Fe}$ layer. A similar suppression of the superconducting proximity effect by a thin ferromagnetic Fe layer was recently observed in $\mathrm{V} / \mathrm{Fe}$ multilayer systems. ${ }^{11,12}$

The resistor circuit corresponding to contact geometry $B$ is illustrated in Fig. 4(b). The equation for the measured resistance using contact geometry $B$ is the same as Eq. (1) derived for contact geometry $A$. Hence, for contact geometry 
$B$, the same analysis can be applied as for geometry $A$. On the other hand, no negative resistance anomaly was observed below $T_{c}$ in resistance measurements performed using contact geometry $C$ (see Fig. 3). This is due to the fact that in this contact geometry, the current and voltage leads are arranged along a straight line, resulting in a single well-defined current path. Consequently, the two voltage probes measure the electrochemical potential along the same current path.

\section{CONCLUSIONS}

Electrical transport measurements were performed on large arrays of 150-nm-wide $\mathrm{V} / \mathrm{Au}$ and $\mathrm{V} / \mathrm{Fe}$ wires using three different contact arrangements. Our results clearly show that a particular arrangement of the electrical contacts in combination with the superconducting proximity effect can induce a pronounced negative resistance anomaly below $T_{c}$. By constructing resistor networks based upon the contact geometries in which such anomalies appear, we were able to derive expressions for the measured resistance. From these expressions we could clearly reproduce the negative resistance anomaly observed below $T_{c}$.

\section{ACKNOWLEDGMENTS}

The authors would like to thank J. M. Kerkhof for his assistance in the preparation of the samples. The $\mathrm{V}$-groove InP substrates used in this study were supplied by Philips Research Laboratories in Eindhoven. This work was partly supported by the Nederlandse Stichting voor "Fundamenteel Onderzoek der Materie (FOM).,
* Present address: Philips Centre for Industrial Technology, P.O. Box 218, 5600 MD Eindhoven, The Netherlands. Email address: j.jorritsma@philips.com

${ }^{1}$ Y. K. Kwong, K. Lin, P. J. Hakonen, M. S. Isaacson, and J. M. Parpia, Phys. Rev. B 44, 462 (1991).

${ }^{2}$ M. Park, M. S. Isaacson, and J. M. Parpia, Phys. Rev. Lett. 75, 3740 (1995).

${ }^{3}$ P. Santhanam, C. C. Chi, S. J. Wind, M. J. Brady, and J. J. Bucchignano, Phys. Rev. Lett. 66, 2254 (1991).

${ }^{4}$ V. V. Moshchalkov, L. Gielen, G. Neuttiens, C. van Haesendonck, and Y. Bruynseraede, Phys. Rev. B 49, 15412 (1994).

${ }^{5}$ B. Burk, C.-J. Chien, V. Chandrasekhar, C. Strunk, V. Bruyndoncx, C. van Haesendonck, V. V. Moshchalkov, and Y. Bruynseraede, J. Appl. Phys. 83, 1549 (1998).

${ }^{6}$ C. Strunk, V. Bruyndonckx, C. van Haesendonck, V. V. Moshchalkov, Y. Bruynseraede, C.-J. Chien, B. Burk, and V. Chandrasekhar, Phys. Rev. B 57, 10854 (1998).

${ }^{7}$ J. M. Graybeal, P. M. Mankiewich, R. C. Dynes, and M. R. Beasley, Phys. Rev. Lett. 59, 2697 (1987).

${ }^{8}$ F. Sharifi, A. V. Herzog, and R. C. Dynes, Phys. Rev. Lett. 71, 428 (1993).
${ }^{9}$ P. Xiong, A. V. Herzog, and R. C. Dynes, Phys. Rev. Lett. 78, 927 (1997).

${ }^{10}$ A. V. Herzog, P. Xiong, F. Sharifi, and R. C. Dynes, Phys. Rev. Lett. 76, 668 (1996).

${ }^{11}$ P. Koorevaar, Y. Suzuki, R. Coehoorn, and J. Aarts, Phys. Rev. B 49, 441 (1994).

${ }^{12}$ J. Aarts, J. M. E. Geers, E. Brück, A. A. Golubov, and R. Coehoorn, Phys. Rev. B 56, 2779 (1997).

${ }^{13}$ R. J. Pedersen and F. L. Vernon, Appl. Phys. Lett. 10, 29 (1967).

${ }^{14}$ M. A. M. Gijs, R. M. Wolf, Th. van Rooy, A. M. Gerrits, M. E. Bijlsma, and A. Gilabert, Solid State Commun. 80, 727 (1991).

${ }^{15}$ J. S. Moodera, L. R. Kinder, J. Nowak, P. LeClair, and R. Meservey, Appl. Phys. Lett. 69, 708 (1996).

${ }^{16}$ R. J. M. van de Veerdonk, J. Nowak, R. Meservey, J. S. Moodera, and W. J. M. de Jonge, Appl. Phys. Lett. 71, 2839 (1997).

${ }^{17}$ A. V. Herzog, P. Xiong, and R. C. Dynes, Phys. Rev. B 58, 14199 (1998).

${ }^{18}$ J. Jorritsma, M. A. M. Gijs, J. M. Kerkhof, and J. G. H. Stienen, Nanotechnology 7, 263 (1996).

${ }^{19}$ S. T. Sekula and R. H. Kernohan, Phys. Rev. B 5, 904 (1972).

${ }^{20}$ J. Jorritsma, Ph.D. thesis, Leiden University, 1999. 